\title{
Approach of Improved Topology Development Protocol In Ad Hoc Network Minimizing The Number of Hops And Maintaining Connectivity of Mobile Terminals Which Move From One To The Others
}

\author{
Lipur Sugiyanta, $\mathrm{PhD}$ \\ Program Studi PTIK \\ Universitas Negeri Jakarta \\ lipurs@unj.ac.id, lipurs@gmail.com
}

\begin{abstract}
Wireless ad-hoc mesh network is a special kind of network, where all of the nodes move in time. Node is intended to help relaying packets of neighboring nodes using multi-hop routing mechanism in order to solve problem of dead communication. Wireless mesh network which engages broadcasting and contains multiple hops become increasingly vulnerable to problems such as routing problem and rapid increasing of overhead packets. During this progress, the delay on account of multi hop characteristics and redundant packets caused by communication nature potentially existed during communication. Typically, delay will increase in linearity with number of hops. There is a certain minimum level of delay that will be experienced due to the time it takes to transmit a packet through a link. Topology development holds a significant point prior to the data transmission. Without improved topology development protocol, this problem can decrease network's performance in overall data transmission. We analyze the delay performance of a multi-hop wireless network with a dynamic route between each source and final destination pair. There are fluctuate interference constraints on the set of links that impose a fundamental delay performance of any instant network topology. At first, we present a similar Link State Routing network simulation to derive such referential lower bounds. We conduct extensive simulation studies to suggest that the average delay of multi-hop transmission policy can be made lower compared to the referential bound by using appropriate functions of network metrics. This paper provides a broadcast framework that engages various network metrics and at the same time maintaining connectivity of nodes (mobile terminals). The framework captures the essential features of the wireless network metrics, i.e. bandwidth, throughput, network buffer, direction, and round trip time. This research is useful since, in many cases, it find that the throughput is the most important parameter in reduction of delay transmission. This result is confirmed with another composite simulation result. Most of network hop delay is impacted with this composite metric, particularly in delay minimization on the longer hops. The reduction achievement on average delay by this algorithm is $0.577 \%$ and the total average delay reduction for this simulated network is $0.683 \%$. This research will be further
\end{abstract}

designed primarily for achieving maximum throughput in the multiple wireless network area.

Keywords-multi-hop; delay; connectivity; metrics

\section{INTRODUCTION}

A Wireless (Ad-Hoc) Mesh Network consists of mobile nodes platforms which are free to move in the area. Node is referred to a mobile device which equipped with built-in wireless communications devices attached and has capability similar to autonomous router. The nodes can be located in or on airplanes, ships, cars, or on people as part of personal handheld devices, and there may be multiple hosts among them. Each node is autonomous. The system may operate in isolation, or have gateways to a fixed network. In the future operational mode, multiple coverage of the network is expected to operate as global "mobile network" connecting to legacy "fixed network".

At each time and every node's positions, a wireless connectivity in the form of a random, single-hop, multi-hop path may exist among nodes. This topology may change as the nodes move or adjust their parameters. Among networks, Wireless (Ad-Hoc) Mesh Network has several characteristics:

1) Dynamic topologies,

2) Bandwidth-constrained,

3) Energy-constrained operation, and

4) Limited physical security.

These characteristics create a set of underlying assumptions and performance considerations for protocol design which extend beyond static topology of the fixed network. The design should react efficiently to topological changes and traffic demands while maintaining effective routing in a mobile networking context. 
All nodes in Wireless (Ad-Hoc) Mesh Network rely on batteries or other exhaustible energy modules for their energy. As a result of energy conservation or some other needs, nodes may stop transmitting and/or receiving for arbitrary time periods. A routing protocol should be able to accommodate such sleep periods without overly adverse consequences. If this is done intelligently, it can utilize network energy and bandwidth resources more efficiently, at the cost of increased route discovery delay. For this network, the design of routing algorithm which able to adapt to the traffic pattern on a demand or need basis is needed for overall network performance.

Routing schemes differ in their delivery semantics: (1) unicast delivers a message to a single specified node; (2) broadcast delivers a message to all nodes in the network; (3) multicast delivers a message to a group of nodes that have expressed interest in receiving the message; and (d) anycast delivers a message to any one out of a group of nodes, typically the one nearest to the source. Broadcast is the dominant form of message delivery on the wireless network.

Research on multi-hop wireless networks have been devoted to routing protocol, system stability, network performance, and throughput maximization. The delay performance of these systems, however, has largely been an open problem. This problem is enormously difficult even in the context of legacy wire networks, primarily because of complex network interactions that complicate the multi-hop routing mechanisms. The paper presents an analysis delay performance network to show an improvement average delay of packet transmission from source to the destination in the dynamic wireless network environment. Furthermore, we re-engineer a routing metric topology development to achieve good delay performance while at the same time maintaining connectivity to other nodes.

In this paper, the proposed framework provides the broadcast schemes in Wireless (Ad-Hoc) Mesh Network that engages various network metrics inside and at the same time maintaining connectivity of nodes (mobile terminals). Various formation options of nodes and their potential overheads and impacts on reduction of delay performance are evaluated via simulation study. We analyze a multi-hop wireless network with multiple source final destination pairs, given routing and traffic information. The remainder of this paper is organized as follows: Section 2 gives preliminaries and our model. Section 3 discusses the detail design of the simulation model, its notations, and assumptions. A performance evaluation of generic algorithms and comparison to a similar Link State Routing broadcast-based network that uses distance weight as the link cost unit are presented in Section 4. Section 5 concludes the paper.

\section{PRELIMINARIES}

Wireless mesh network is generally set up with a centralized access point for provide high level of connectivity in certain area. The access point has knowledge of all devices in its area and routing to nodes is done in a table-driven manner [1][2][5]. Nemoto introduced a technical review of wireless mesh network technology products that implemented IEEE802.11 standard through experiments of fixed wireless mesh network nodes [2]. In terms of review the network performance at this stage, it will be represented as the view of use and evaluation of outdoors Muni-WiFi devices in accordance to applying the legacy LAN technology inside the corporate network. Performance of network access layer, i.e. performance of voice and TCP data transmission in terms of throughput, response time between mesh nodes, and communication delay in multi-hop transmission are presented.

However, Nemoto intended to operate in static topology network [2]. With recent performance in computer and wireless communications technologies, advanced mobile wireless is expected to see increasingly widespread use and application. The vision of future mobile ad hoc networking is to support robust and efficient operation in mobile wireless networks by incorporating routing functionality such that networks are capable to be dynamic, rapidly-changing with random, multihop topologies which are likely composed of relatively bandwidth-constrained wireless links. Supporting this form of host mobility requires address management, protocol interoperability enhancements and the likes.

Broadcasting plays a critical role especially in vehicular communication where a large number of nodes are moving and at the same time sending a large size of packet. Broadcast is essential in order to either transmit packet data or build topology prior to data transmission. In wireless network where nodes communicate with each other using broadcast messages, the broadcast environment works as receivers collect information from all transmitting nodes within its coverage pattern's neighborhood, and then allowing receivers to aware of immediate surrounding respond before re-transmitting packet. Several transmissions may be unnecessary during broadcasting mechanism. These redundant cause the broadcast storm problem [8], in which redundant packets cause contention and collision consume a significant delay of the aimed transmission time. Thus, routing protocols should be capable to respond these changes using minimum signaling and taking into account the routing topology metric as a parameter distributed in the network. To address these challenges, a delay-efficient routing must satisfy the following properties; (1) ensure high throughput and (2) allocate resources equitably. Multiple link hop paths in the network have to be scheduled such that selected link paths are not starved for service. Starvation leads to an increase in the average delay in the network.

Minimizing the delay involves applying a path selection mechanism of routing metric to multiple routes, in order to select (or predict) the best route. The metric is computed by a routing algorithm, and can cover such information as throughput, round trip time, hop count, interface buffer, load, MTU, direction, and communication cost. The routing table stores the best possible routes and other potential routes.

\section{SIMULATION MODEL, NOTATIONS, AND ASSUMPTION}

Relays are intermediate nodes between source and final destination which help relaying packets using multi hop routing mechanism. The appearance of relays is required to avoid dead communication if the distance is not in the proximity of each node. It can extend the mobility and expand the coverage area, but in the same time increase the delay time. In general, the more relay nodes (hop), the longer the delay time. Energy consumption is also affected. Direct transmission is seemed to have more aggregate energy required than indirect 
communication. Thus, the adjustment of relay nodes will influence the balance of delay time and energy consumption.

The proposed framework assumes that nodes are capable of dynamically adjusting their relay nodes on per move step base. It attempts to minimize the number of relay nodes between source and final destination pairs and at the same time maintain the node's energy level required. This behavior is almost similar to MANET routing protocols (e.g., AODV, DSR and TORA), One common property of these routing protocols is that they discover routes using broadcast flooding protocols whose value of distance metric in order to minimize the number of relay nodes between any source and final destination pair.

The approach is initiated from broadcast mechanism and propagated through node-to-node based routing topology metrics approach. During propagation, it takes into account all topology development, route discovery, and data transmission. Each source injects single big packet which fragmented into multiple packets in the network, which traverse through the network until those reach the final destination. Packets are queued at each node in its path where it waits for an opportunity to be transmitted. This model is not only applicable in direct communication (one hop transmission) but it can also work in multi-hop transmission. In this situation, when the source and final destination nodes are located outside the maximum transmission range, source node is capable to discover multiple hop routing efficiently thus maintain the energy level required in comparison to standard flooding based ad hoc routing designs.

\section{A. The Model}

Simulation describes that antenna module installed in each node is capable of dynamically adjusting the transmission energy used to communicate with other nodes. Industrial standard of antenna module supports a management for controlling this energy consumption. Simulation assumes that the energy consumption required to transmit a packet between nodes $\mathrm{A}$ and $\mathrm{B}$ is similar to that energy required between nodes $\mathrm{B}$ and $\mathrm{A}$ if and only if the distance and the size of packet are same. The coverage distance range of the nodes is a perfect symmetric unit disk (omni-directional). If $d_{x, y} \leq r_{x} \rightarrow A$ and $B$ can see each other.

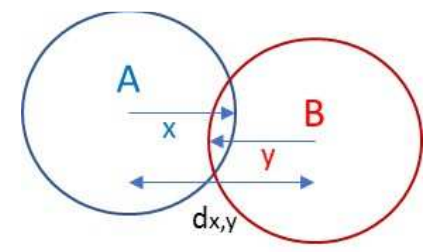

This assumption may be acceptable in the condition that interference in both directions is similar in space and time; which is not always the case. Usually interference-free Media Access Control (MAC) protocol such as Channel Sense Multiple Access (CSMA) may exist. In addition, wireless link channel is assumed to have no physical noise; i.e., the errors in packet reception due to fading and other external interferences are not considered as a serious problem. Packets from sender to receiver will be transmitted as long as the bandwidth capacity is sufficient and the received signal to noise ratio (SNR) is above a certain minimum value. Thus, every packet successfully received is acknowledged at the link layer and de-encapsulate at the higher layer. Each node is capable of measuring the received SNR by analyzing overheard packet. A constant bit error rate (BER) is defined for the whole network. Whenever a packet is going to be sent, a random number is generated and compared to the packet's CRC. If the random number is greater, the message is received, otherwise it is lost. The default value for the BER is 0 , which means there is no packet loss due to physical link error.

Simulation cover a single area of homogeneous nodes that communicate with each other using the broadcast services of IEEE 802.11. There are nodes with different roles simulated in this simulation, namely initiator node/source node, receiver node, sender node, destination node, and final destination node. Initiator node/source node is node that initiates transmission of packet. Packet can be either route discovery or data transmission. Like other nodes, initiator is always moving with random direction, speed, and distance. At the time it is moving, initiator node is always sensing its neighbor to maintain connectivity. Receiver node is node that can be reached by source/sender node. Nodes are defined as neighbors if it located within its distance radius range. At initial time, node senses its neighbors before packet data is required to be transmitted. Coverage neighbor nodes always receive packets that are broadcasted from sender. Destination node is selected receiver node in multi hop transmission that should relay packets to the next receiver node. Final destination node is node that became the end destination of packets.

The layered concept of networking was developed to accommodate changes in local layer protocol mechanism. Each layer is responsible for a different function of the network. It will pass information up and down to the next subsequent layer as data is processed. Among the seven layers in the OSI reference model, the link layer, network layer, and transport layer are 3 main layers of network. The framework is configured in those layers. Genuine packets are initiated at Protocol layer, and then delivered sequentially to next layer as assumed that fragmented packets to be randomly distributed. Simulation models each layer owned with finite buffers. Limited buffer makes packets are queued up according to the drop tail queuing principle. When a node has packets to transmit, they are queued up provide the queue contains less than $K$ elements $(K \geq 1)$. To increase the randomization of the simulation process, simulation introduces some delay on some common processes in the network, like message transmission delay, processing delay, time out, etc. This behavior will result that at each instance of a simulation would produce different results. The packets exchanged between sender and receiver is of a fixed rate transmission $\lambda$ based on a Poisson distribution. Nodes that have packet queued are able to transmit it out using in each available bi-directional link channel.

Energy is power kept in each node. Heinzelman et al. assumed that the radio dissipates $E_{\text {elec }}=50 \mathrm{~nJ} / \mathrm{bit}$ to run the transmitter or receiver circuitry and $\varepsilon_{\text {amp }}=100 \mathrm{pJ} / \mathrm{bit} / \mathrm{m}^{2}$ for the transmit amplifier [6]. Thus, to transmit a k-bit message a distance $\mathrm{d}(\mathrm{m})$ using this radio model, the radio expends:

$E_{T X . B i t}(k, d)=E_{\text {elect }} * k+\varepsilon_{\text {amp }} * k * d^{2}$

and to receive this message, the radio expends: 
$E_{R X}(k)=E_{\text {elect }} * k$

The energy model included in simulation was based on the following formulas, taken from [5]:

$E_{\text {TX.Bit }}=E_{\text {elect }}+\left(\varepsilon_{\text {amp }} * \pi r^{2}\right)$

$E_{\text {RX.Bit }}=E_{\text {elect }}$

Notations explain:

$E_{T X . B i t}(k, d)$ : energy needed to transmit message consists of $\mathrm{k}$ bit along distance d,

$E_{R X}(k)$ : energy needed to receive message consists of $\mathrm{k}$ bit,

$k$ : number bits of message,

$d$ : distance $(\mathrm{m})$,

$r$ : radius of node coverage $(\mathrm{m})$.

The energy behaviors of node are defined as follow:

- During the idle time, a node does not spend energy. Even though this assumption has been proven untrue because being idle might be as costly as receiving data, this is still an assumption that can be done in most experiments, since the most important factor is the overhead in terms of message exchange and its associated cost.

- The nodes are assumed to have one radio for general messages. The main radio is used in all operations when the node is in active mode, and to send and receive control packets. When this radio is turned off, then no messages will be received and no energy will be used.

- Energy distribution among nodes can either be constant value, normally distributed, Poissonly distributed, or uniformly distributed.

\section{B. Topology Development Algorithm}

The goal of this research is to find out the essential metric of wireless network parameters which required during topology development prior to data transmission in the network and maintaining connectivity to the others. The parameters analyzed are distance, round trip time, interface buffer/network buffer, throughput, energy, and combination of those.

The core algorithm is developed from static mode (e.g., sensor networks). The enhancement algorithm for serving mobility then detailed in support of topology building, topology maintenance, and routing maintenance. We show our methodology on a tree network. The tree topology decomposes the paths between source and final destination into several route paths. The algorithm underestimates the interference between the route paths. The algorithm starts to operate with building the network topology. Establishment of the next hop in this multihop environment is conducted with topology development mechanism. The role of the route maintenance algorithm is to make sure that a minimum flow of packets is transmitted in order to maintain the route when there are no data packets available to send at the transmitter.
Network topology must be executed before data transmission takes place. Topology development is proactive, it uses Topology Control (TC) messages to discover and disseminate link state information. It involves transmit and receives of HELLO packets, REPLY packets, CONFIRM packets, and so on; mostly redundant. These are packets that successfully received by link layer and will update an entry in the neighbor table which cache information about surrounding nodes exists. HELLO packets and corresponding REPLYs have contents of [ID, hop, energy, time, initialTime], where ID is a unique neighbor node (IP address), hop is a number which increment each time packet reach at relay node, energy is current available energy level needed to ensure the communication with the neighbor node, time is current time at which this event is executed, and initialTime is time from which this event was generated.

The routing maintenance algorithm is responsible for performing the route optimization operation that leads to the discovery of routes changes. The route maintenance algorithm performs two basic operations: initiate broadcast maintenance packets, which computes whether a route optimization between two nodes is needed and sets up broadcast mechanism; and executes maintenance packets, which determines when to transmit routing maintenance packets. The framework optimizes routes through sequence of steps to converge to an optimum route. The step refers to the event in which a packet initiates a source node to transmit a Hello request for the first time. The network will converge as fast as the transmission speed of data transmitted by node.

We built network simulator to evaluate this performance. The simulator supports physical, link and routing layers for single/multi hop ad-hoc networks. We assume that IEEE 802.11 Distributed Coordination Function (DCF) or MAC protocol which uses Channel Sense Multiple Access with Collision Avoidance (CSMA/CA) already deployed. Successfully received packet by receiver's interface is packet whose SNR is above a certain minimum value otherwise the packet cannot be distinguished from background noise/interference. Packets are transmitting through physical layer in accordance with Poisson distribution. Communication between two nodes in IEEE 802.11 uses RTS-CTS signaling before the actual data transmission takes place. Simulation simulates this with random hearing to link's condition.

The simulator uses two-steps propagation model to simulate interactive propagation in the operation of the protocol in dynamic environment. As a future research, the appropriate propagation model that best matches to this environment should replace the simple two-steps model presented here. The twosteps propagation model is appropriate for outdoor environments where a line of sight communication existed between the transmitter and receiver nodes and when the antennas are omni-directional.

At first, when a node first starts, it only knows of its immediate neighbors, and the direct cost involved in reaching them. (This information, the list of destinations, the total cost to each, and the next hop to send data to get there, makes up the routing table, or distance table.) Each node, on a regular basis, sends broadcast packets to neighbors to get all costs of 
destinations. The neighboring node(s) examine this information, and compare it to what they already 'know'; anything which represents an improvement on what they already have, thus update their own routing table(s). Over time, all the nodes in the network will discover the best next hop for all destinations, and the best total cost. When one of the nodes involved goes down, those nodes which used it as their next hop for certain destinations discard those entries, and create new routing-table information. They then pass this information to all adjacent nodes, which then repeat the process. Eventually all the nodes in the network receive the updated information, and will then discover new paths to all the destinations which they can still "reach".

During this sequence, relay node is determined by relevant information gathered from neighbor nodes. After omitted redundant packets and based on calculation metric value, relay node is set (i.e., a small set of nodes that potentially forward the broadcast packet) to achieve high delivery ratio with certain metric consideration. This is the second step. It means that only selected neighbors able to forward the packet to the next neighbors. The selected neighbor or new relays added to a route during iteration are very much dependent on the relay found in the previous iteration. The set can be selected dynamically (based on both topology and broadcast state information). This relay node set forms a connected dominating set (CDS) and achieves full coverage of connected network. It is possible that the first iteration, which seemed as most optimum value of metric value is not the route achieving the optimum topology with optimum delay path.

Real data transmission is triggered by source node which injects one packet into the protocol layer. The packets either fragmented or not, flow through node layers at every time-slot. The length of the active periods (denoted by random variable) is distributed randomly according to Mersenne Twister algorithm. The mean of transmission rate and arrival rate of packets can be controlled by changing the value of $\mathrm{p}$, a Poisson distribution. Upon receiving a packet, neighbors create reply packet which contain its condition (i.e. trip time, interface buffer) and sent it back to sender. The arrival process is defined as the arrival packets stream at each node is a series of active and idle periods. The received packet is then processed by the layering module with the result that one of the following actions is taken: (i) the packet is passed to the higher layers if both MAC and IP addresses match; (ii) the packet is dropped if neither MAC nor IP addresses match; or (iii) the packet is forwarded to another node when only the MAC address matches. In the latter case, it searches the routing table to find the next route node with the higher metric calculation to reach next destination node. When receiver receives a packet data from the higher layers it searches the routing table to see if a route toward the destination node exists. If this is not the case, node searches the neighbor table to see if information regarding the destination node is available. After the neighbor node replies with a packet of its own then route optimization follows as described previously. When nodes are mobile and no data packets are available for transmission, a source node required to transmit explicit signaling packets to maintain a topology.

Because several relay nodes may exist between source and final destination, the source node will choose the one providing a highest metric value. Multiple packets are sent to that single (next) relay node. From the simulation, it noted that transmission of multiple route-redirect packets wastes bandwidth and network resources (overhead packets increased). For sparsely populated networks, this may not be a problem. However, this is an issue in the case of densely populated networks where several potential nodes can be chosen. The framework addresses this issue by giving priority for the execution of an update routing maintenance packet to the potential neighbor node that computes highest route metric energy-distance values first. After receiving an update routing maintenance packet, a node modifies its routing table, putting the source of the received packet as the next hop node for the specific sender-destination route path. To execute preferential event in sequentially distributed events, we used a simple approach that consists of applying a different timeevent execution after the triggering event takes place. The lower and upper bound of the queuing interval are set such that they do not interfere with predefined timers used by the other events for layers and modification events.

\section{PERFORMANCE EVALUATION}

In this section, an evaluation of the framework is discussed and followed by a number of performance issues associated with routing metrics and route maintenance. Much of the analysis for multi-hop wireless networks has been limited to establishing the performance of the network. In this paper, we have taken an important step towards the expected delay analysis of network.

The general research on the delay analysis has progressed in the following main directions:

1) Heavy traffic using fluid models: used to either establish stability of the system or to study the workload process in the heavy traffic environment.

2) Large Deviations: to calculate queue-overflow probability.

Here, we have taken a different approach to reduce the wireless network to single topology tree network which are then analyzed to construct the delay performance for data transmission. This technique captures the essential features of the wireless network and is useful since, in many cases, we can also find that the proposed framework performs higher than the similar Link State Routing (LSR) network. The similar LSR network is selected because it is simple to deploy and can be used for analyzing a large scale of packets processes using known network topology. We compare the framework and similar LSR network to best understand the various tradeoffs and limitations of the algorithm.

A similar (LSR) network would generate full routing tables in advance where, all nodes in the network would be aware of distance level and routes to all other nodes in the network. This network can compute the optimum metric with shortest distance to a next relay node by listening replies of topology construction and topology maintenance packets transmitted by the neighbors. This network operation requires each node in the network to broadcast a routing packet. The broadcast packets contain information about the distance metric of all known destinations. Each node floods the network with information about what other nodes it can connect to, and the received packets may require to be forwarded by other nodes to propagate the entire network. 
After collecting packets from all nodes of the network, any node should be capable of computing optimum routes to any other node in the network. Each node then independently assembles this information into a tree. Using this tree, each node then independently determines the least-cost path from itself to every other node using a standard shortest paths (distance) algorithm. The iteration of propagation events to be entirely flooded mainly depends on the density of nodes in the network. The result is a tree rooted at the source node such that the path through the tree from the root to any other node is the least-cost path to that node. This tree then serves to construct the routing table, which specifies the best next hop to get from the current node to any other node.

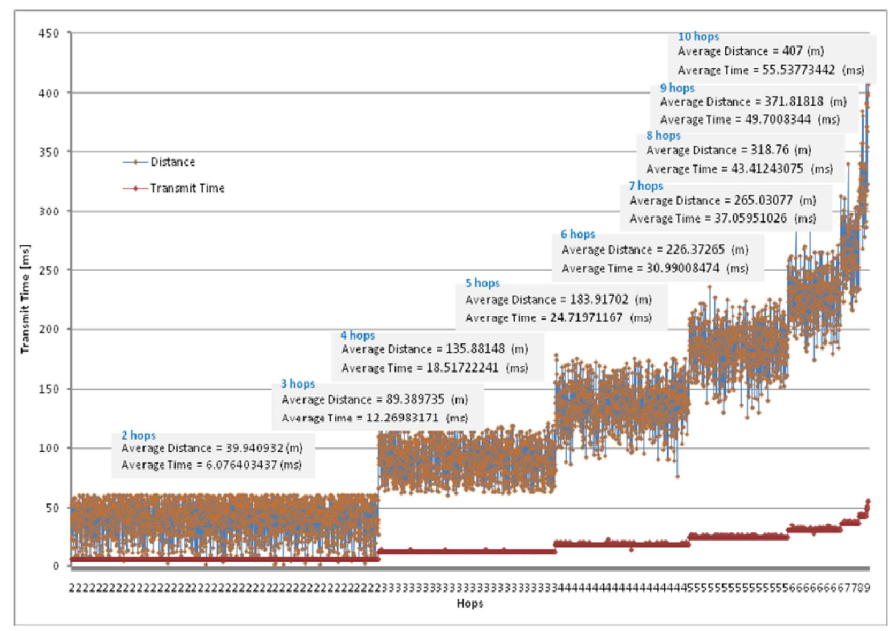

Fig. 1. The delay performance of similar LSR network.

We consider a network composed of 20 nodes located within transmission range of each other. In this example, we analyze the wireless tree topology with randomly generated flows initially described in Section 3. There are several metrics in this system which investigated with each topology development under the 20 nodes network model. We studied the network for several scenarios with different initiator nodes load. We find that depending on the input topology metric, the algorithm computes different hop/relay connectivity type for the packets flow in the system. We discuss five representative topology metrics to evaluate the impact of the topology development made in the analysis. The mean arrival rate of fragmented packets of completed 10000 bytes packet, a Poisson distribution, is set to 100 .

\section{A. Case 1}

For the given round trip time metric weight, the algorithm computes the decomposition of topology into maximum 10 tree hops. $37.37 \%$ is two hops, $21.98 \%$ is three hops, $17.68 \%$ is four hops, $12.71 \%$ is five hops, and rest is for other hops. Note that this metric impacts on the number of hops from two to four significantly, but this effect is not resulting in larger delay minimization on the longer hops. Also, note from Fig. 2 that the average distances for different hops are almost similar. The higher reduction on average delay by this metric algorithm is $0.183 \%$ compared with Fig. 1 respectively. The total average delay reduction for this simulated network is $0.344 \%$.

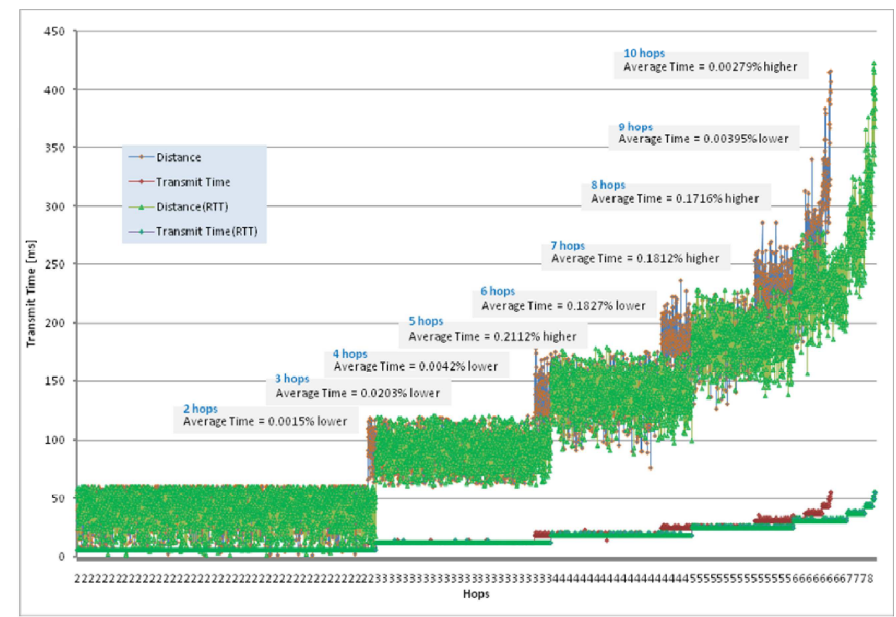

Fig. 2. Comparison between improved Topology construction with RTT metric and similar LSR network.

\section{B. Case 2}

For the given throughput, metric weight, the algorithm computes the decomposition of topology into maximum 12 tree hops. $37.25 \%$ is two hops, $21.58 \%$ is three hops, $18.57 \%$ is four hops, $12.88 \%$ is five hops, and rest is for other hops. Almost each hop delay is impacted with this metric from two to eight hops, but this effect is not resulting in larger delay minimization on the longer hops. Also, note from Fig. 3 that the average distances for different hops are almost similar, except for 11 and 12 hops. The higher reduction on average delay by this metric algorithm is $0.359 \%$ compared with Fig. 1 respectively. The total average delay reduction for this simulated network is $0.542 \%$.

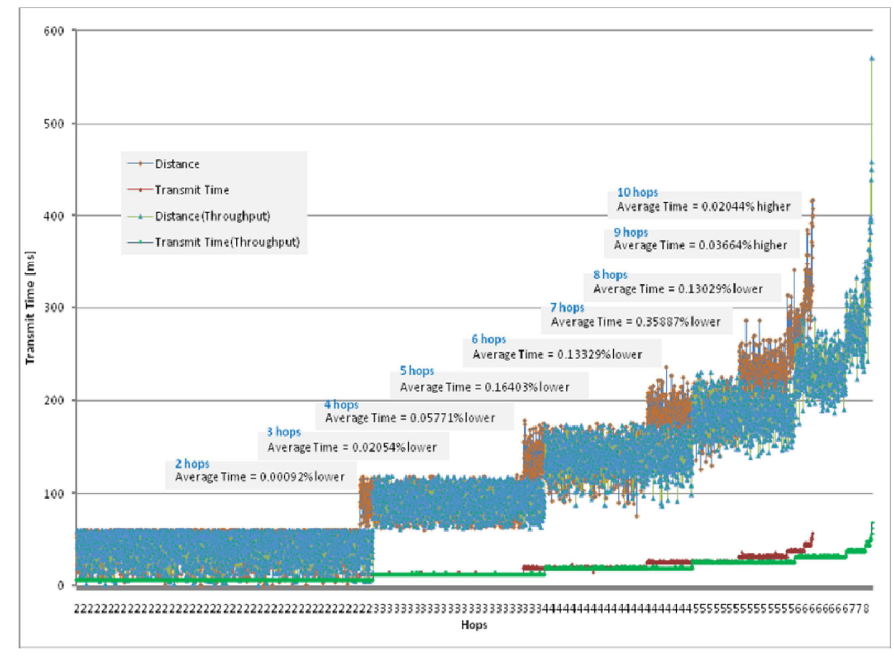

Fig. 3. Comparison between improved Topology construction with Throughput metric and similar LSR network.

\section{Case 3}

Direction can be distinguished as approaching and away. For the given direction, metric weight, the algorithm computes the decomposition of topology into maximum 10 tree hops. $37.22 \%$ is two hops, $22.32 \%$ is three hops, $18.91 \%$ is four hops, $12.58 \%$ is five hops, and rest is for other hops. Only small portion of network delay is impacted with this metric, particularly from five to seven hops, but this effect is not resulting in delay 
minimization on the shorter hops. From Fig. 4, the average distances for different hops are almost similar. The higher reduction on average delay by this metric algorithm is $0.388 \%$ compared with Fig. 1 respectively. The total average delay reduction for this simulated network is $0.522 \%$.

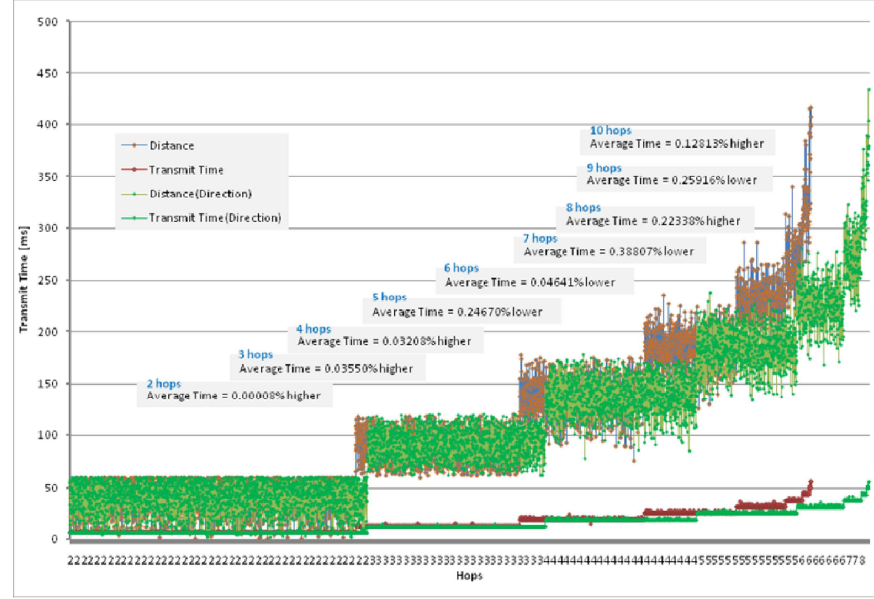

Fig. 4. Comparison between improved Topology construction with Direction metric and similar LSR network.

\section{Case 4}

Cmbination of distance metric and energy metric is used. For the given metric weight, the algorithm computes the decomposition of topology into maximum 12 tree hops. $36.18 \%$ is two hops, $22.01 \%$ is three hops, $18.13 \%$ is four hops, $11.65 \%$ is five hops, and rest is for other hops. With the average distances for different hops is almost similar, less portion of network delay is impacted with this metric as shown in Fig. 5. The higher reduction on average delay by this metric algorithm is $0.253 \%$ compared with Fig. 1 respectively. The total average delay reduction for this simulated network is almost zero.

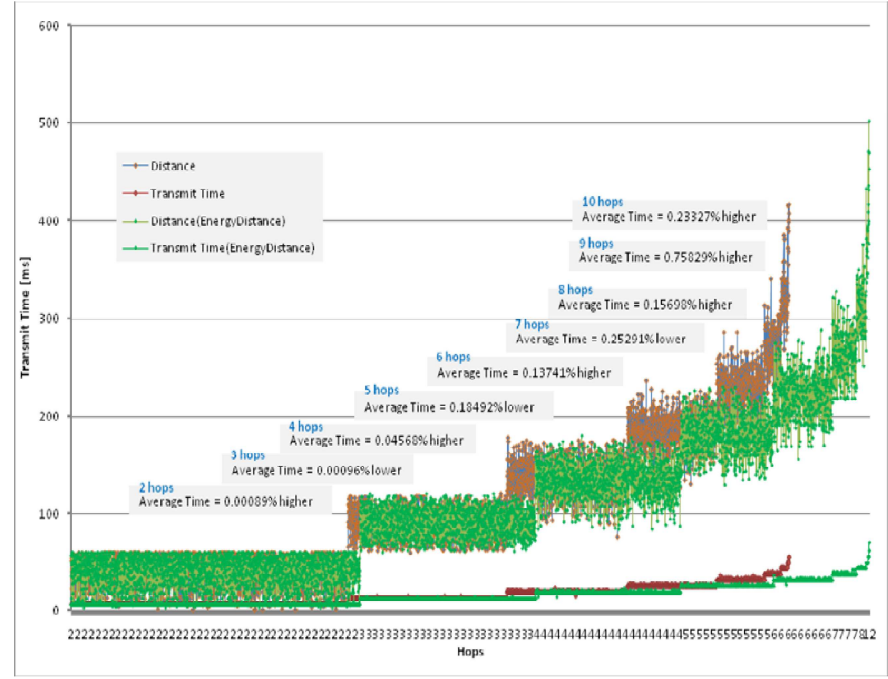

Fig. 5. Comparison between improved Topology construction with Energy+Distance metric and similar LSR network.

\section{E. Case 5}

A composite metric consists of distance, energy, interface buffer, round trip time, and direction is set. For this given metric weight, the algorithm computes the decomposition of topology into maximum 10 tree hops. $36.56 \%$ is two hops, $21.46 \%$ is three hops, $18.02 \%$ is four hops, $12.18 \%$ is five hops, and rest is for other hops. Most of network hop delay is impacted with this metric, particularly in delay minimization on the longer hops. From Fig. 6, the average distances for different hops are almost similar. The higher reduction on average delay by this metric algorithm is $0.577 \%$ compared with Fig. 1 respectively. The total average delay reduction for this simulated network is $0.683 \%$.

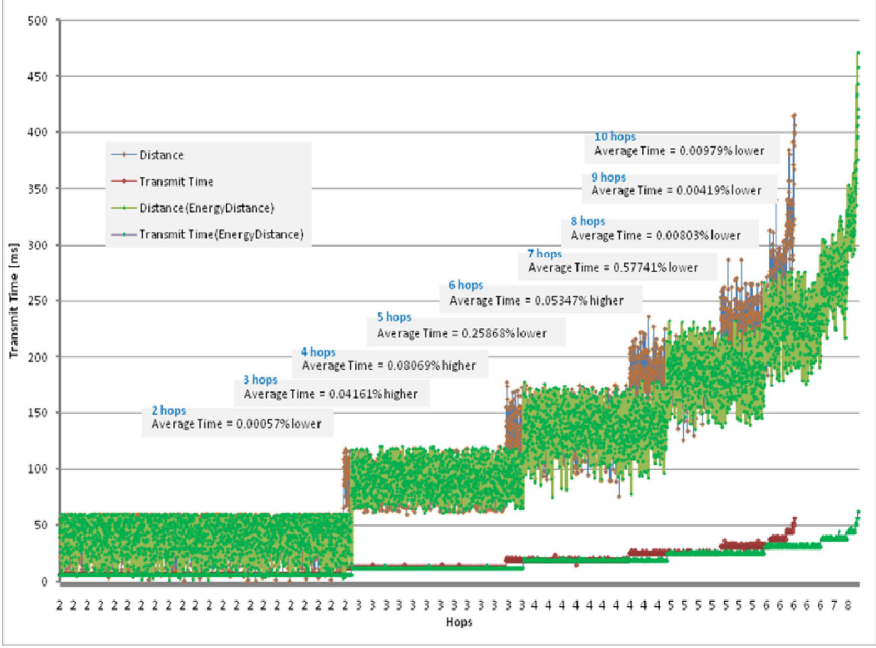

Fig. 6. Comparison between improved Topology construction with Composite metric and similar LSR network.

\section{CONCLUSION}

In this paper, we have described a simple approach to reduce the delay in a multi-hop wireless topology construction routing prior to carry out the data transmission analysis. The analysis is taken generally and admits a large class of arrival processes. The analysis already includes handling multiple topology type combination. The main difficulty however is in identifying the bottlenecks in the network. The result obtained in this simulation is compared against the similar LSR network with the initial average distances for different hops are kept similar. The delay performance in similar LSR is important to identify reference optimum relay hop delay and help in the design of a delayefficient policy of comprehensive network simulation. Here, we have taken a different approach to reduce the wireless network delay in multi hop environment through the analysis of network routing metric in charge of the topology creation. This algorithm captures the essential wireless network performance parameters, i.e. bandwidth, throughput, network buffer, direction, and round trip time. This result is useful since, in many cases, we can also find that the throughput is the most important parameter in reduction of delay transmission. This result is confirmed with another simulation result using composite network metric. It is interesting to note that the routing metric policy, which was designed primarily for achieving maximum throughput in the single wireless network area, can also be engineered to achieve good delay performance in multiple wireless network area. 


\section{ACKNOWLEDGMENT}

The authors would like to thank the anonymous reviewers for the helpful comments and suggestions.

\section{REFERENCES}

[1] Masato, Tsuru. "Simulation-based Evaluation of TCP Performance on Wireless Networks". Journal of the Japan Society for Simulation Technology: 67-73, 2009.

[2] Nozomu, Nemoto. "Consideration and Evaluation of Wireless Mesh Network". Nomura Research Institute (NRI) Pacific Advanced Technologies Eng.: 70-85, 2006.

[3] Article in a conference proceedings:
[4] Javier G., Andrew T. C., Mahmoud N., Chatschik B. "Conserving Transmission Power in Wireless Ad Hoc Networks". Network Protocols Ninth International Conference on ICNP: 24-34, Nov 2001

[5] Chang-Woo Ahn, Sang-Hwa Chung, Tae-Hun Kim, Su-Young Kang. "A Node-Disjoint Multipath Routing Protocol Based on AODV in Mobile Adhoc Networks". In: Proceeding of Seventh International Conference of Information Technology ITNG2010: 828-833, April 2010.

[6] Prasanthi. S, Sang-Hwa Chung. "An Efficient Algorithm for the Performance of TCP over Multi-hop Wireless Mesh Networks". In: Proceeding of Seventh International Conference of Information Technology ITNG2010: 816-821, April 2010.

[7] Heinzelman, W., Chandrakasan, A., Balakrishnan, H. "Energy-efficient communication protocol for wireless microsensor networks". In: Proceedings of the 33rd International Conference on System Sciences (HICSS): 1-10, 2000.

[8] A. Feldmann, N. Kammenhuber, O. Maennel, B. Maggs, R. D. Prisco, and R. Sundaram. "A methodology for estimating interdomain web traffic demand”. In IMC, 2004. 